\title{
LA ENERGÍA EÓLICA EN ESPAÑA
}

\author{
Cayetano Espejo Marín \\ Departamento de Geografía Física, Humana y Análisis Regional \\ Universidad de Murcia
}

\section{RESUMEN}

Desde mediados de los años noventa se ha producido en España una expansión extraordinaria del aprovechamiento de la energía eólica para la generación de electricidad. La disponibilidad de zonas con potencial eólico estimable junto con las políticas de apoyo a las energías renovables, sobre todo a la eólica, han contribuido a un enorme desarrollo industrial y tecnológico de este sector. España es líder mundial, tanto en potencia instalada, donde ocupa el tercer puesto detrás de Alemania y Estados Unidos, como en número y volumen de empresas que participan en los distintos ámbitos de la energía eólica. En este artículo se analizan las características del sector eólico español.

Palabras clave: viento, aerogenerador, energía eólica, electricidad, impacto ambiental.

\section{ABSTRACT}

From the mid-1990s Spain has seen an extraordinary growth in the exploitation of wind energy for generating of electric power. Both, the existence of areas which are potential sources of this type of energy and the development of policies supporting renewable forms of energy, maily wind one, are the main factors that have promoted the industrial and technological development of this sector. Spain is the third country, just behind Germany and the USA, as regards installed power and it is among the leading countries in the world regarding the number of companies connected to the sector. In this article I will analyse the main characteristics of the production of wind energy in Spain.

Key words: wind, wind-driven generator, electricity, impact on the environment.

\section{Introducción}

España es uno de los países del mundo que ha experimentado un mayor desarrollo industrial y tecnológico en el sector eólico dedicado a la producción de electricidad. La potencia instalada ha pasado de tener un carácter testimonial a comienzos de los años 
noventa, con una potencia instalada que no llegaba al medio centenar de megavatios (MW), a una cifra próxima a los cinco mil a mediados de 2003. En la actualidad España se sitúa en el tercer lugar a nivel mundial en potencia instalada, detrás de Alemania y Estados Unidos. Este desarrollo ha sido posible por la disponibilidad de zonas con estimable potencial eólico, por las políticas de fomento de las energías renovables y por el interés despertado en las empresas que trabajan en este sector. Según la Plataforma Empresarial Eólica, trescientas compañías participan actualmente en la industria eólica española, dedicadas a la fabricación de componentes y equipos, promoción y explotación, construcción y servicios, transporte y distribución.

La Ley 54/1997, de 27 de noviembre, del Sector Eléctrico, en su artículo 27 define el «Régimen Especial de la Producción Eléctrica», que incluye las energías renovables. Este régimen regulador específico constituye un evidente apoyo público a las energías renovables, siempre que cumplan unas condiciones mínimas en términos de eficiencia. Las ventajas de estar acogido al régimen especial de producción son dos: garantía de venta de la energía producida, al estar obligadas las compañías distribuidoras de la zona de referencia a adquirir la producción de estas instalaciones, y en segundo lugar la percepción de una remuneración adecuada por la energía producida.

\section{El viento como recurso para la producción de electricidad}

La energía eólica se aprovecha mediante la transformación de la energía cinética del viento en energía eléctrica a través de aerogeneradores, que utilizan una hélice para transmitir el movimiento que el viento produce en sus palas al rotor de un alternador. Cuando una instalación eólica necesita producir electricidad para verter a la red de distribución se agrupan varios aerogeneradores, dando lugar a los denominados parques eólicos. Si su objetivo es suministrar electricidad a puntos de consumo aislados (viviendas dispersas, granjas, explotaciones agrícolas,...) se utilizan equipos de pequeña potencia, en general de varias decenas de kilovatios $(\mathrm{kW})$.

La energía eólica para que pueda ser utilizada con cierta eficacia en una zona determinada, las características del viento deben cumplir una serie de condiciones relativas a velocidad, continuidad, estabilidad, etc. Un dato de gran importancia al respecto es la «densidad de potencia» del viento, es decir, el valor máximo de la potencia que se puede conseguir por cada unidad de área barrida por el viento. En concreto, por debajo de los 50 vatios $/ \mathrm{m}^{2}$ no tiene interés el emplazamiento de instalaciones eólicas; y sólo por encima de $\operatorname{los} 200$ vatios $/ \mathrm{m}^{2}$ comienzan a resultar auténticamente rentables. Aún así hay que tener en cuenta que hacen falta densidades de potencia del viento superiores a $1.000 \mathrm{vatios} / \mathrm{m}^{2}$ para que los aerogeneradores tengan un rendimiento aceptable (Unesa, 1998).

Según J. Olcina (1994), dejando al margen los sectores montañosos donde el venteo es continuo y si acontecen ventoleras más intenso, en España los sectores más afectados por los vientos a gran velocidad tienen una distribución anárquica y ello está en función de disposiciones de línea de costa y situación de relieves próximos proclives a la formación de pasillos o ventanías. Por tanto es posible distinguir:

- Litoral cantábrico y dentro de él la costa gallega entre la Ría de Arosa y la Estaca de Bares, y el sector ovetense en torno al Cabo de Peñas.

- Valle del Ebro, con un área de mayores velocidades del viento alrededor de la capital zaragozana.

- Castilla y León, en una franja extendida entre el suroeste de Burgos y noreste de Salamanca. 
- La Mancha, y en particular la provincia de Albacete.

- Litoral mediterráneo con algunas ventanías: litoral gerundense (El Ampurdán), litoral levantino (entre Valencia y el Cabo de San Antonio) y la franja litoral de la Sierra del cabo de Gata (Almería).

- El cuadrante suroccidental de España, entre las bahías de Algeciras y Cádiz.

Varios son los factores que explican la presencia constante de vientos en algunas zonas de la Península Ibérica.

Galicia, por su situación geográfica, constituye una de las principales vías de entrada a Europa de las profundas borrascas y sistemas frontales formados sobre el Océano Atlántico, en el espacio frontera entre las masas de aire frío polar y las masas de aire cálido de origen tropical. La existencia de fueres gradientes de presión ligados a estas áreas depresionarias generan fuertes vientos (Martí, 1998).

El viento es un elemento de particular importancia en el Valle del Ebro dada la frecuencia con que se observa. Su encuadramiento entre dos macizos montañosos de considerable altitud forma un pasillo de dirección casi obligada para el viento, que describe una trayectoria noroeste-sureste o al contrario, recibiendo en cada caso la definición de cierzo o bochorno. El viento sopla de forma continua e intensa en Zaragoza. Las calmas suponen sólo un 13,5\% anual. La velocidad es particularmente alta durante el invierno y la primavera (Hernández, 1990).

Tarifa es el enclave andaluz y de España que menos días de calma registra, tiene una media anual en porcentaje de frecuencias de 2,7 \% (Viedma, 1998). En Almería el viento constituye uno de los factores climáticos más importantes de la provincia. El relieve Bético, debido a su configuración y orientación, canaliza los flujos aéreos, y éstos tienden a adaptarse al terreno buscando los puntos más bajos (Viedma, 1983).

Uno de los rasgos desde el punto de vista climático que distingue a las Islas Canarias es el dominio, casi constante, de un cinturón de altas presiones dinámicas que da lugar a una persistencia de los vientos alisios. Estos vientos, procedentes del flanco oriental del anticiclón de las Azores, poseen una gran regularidad anual, aunque con cierto predominio en la estación de verano (Martínez de Pisón, 1987). La regularidad del régimen de los alisios y el hecho de que en los lugares abiertos a estos vientos la frecuencia de las velocidades comprendidas entre los 25 y $40 \mathrm{~km} / \mathrm{h}$ se acerque al $50 \%$, junto a la poca frecuencia de los superiores a $50 \mathrm{~km} / \mathrm{h}$ y a que el recorrido medio del viento sea del orden de $20 \mathrm{~km} / \mathrm{h}$, hace que estos lugares resulten idóneos para la utilización de la energía eólica (Font Tullot, 2000).

\section{Antecedentes de la producción de electricidad de origen eólico}

Como precursor de los actuales aerogeneradores es la aeroturbina de Lacourt (1892), máquina de cuatro palas de 25 metros de diámetro capaz de desarrollar entre 5 y $25 \mathrm{~kW}$. Durante las primeras décadas del siglo XX, el avance de la tecnología aeronáutica establece las bases de los actuales aerogeneradores, aunque es necesario esperar hasta la crisis del petróleo de 1973 para que la energía eólica cobre un vivo impulso como fuente energética para la generación de electricidad (Alonso, 1993).

La posibilidad del aprovechamiento de la energía del viento en España con fines energéticos se remonta a mediados de los años cincuenta del siglo pasado. En 1955 se publica el informe La energía del viento y su aprovechamiento, del que son autores J.A. Barasoaín y L. Fontán. De ambos también es el estudio Prospección eólica y solar en España (1962). Dos décadas más tarde J.L. Cardona (1981) publica su estudio Energía 
eólica y aeroturbinas. Posibilidades de utilización en España. En el tercer capítulo hace un somero análisis del viento en España, comenta el mapa realizado en 1961 por J.A. Barasoaín y L. Fontán, de velocidad media del viento en $\mathrm{m} / \mathrm{seg}$., y pone de manifiesto como zonas con características importantes: el extremo Noroeste Peninsular, el Valle del Ebro, la zona del Estrecho de Gibraltar, algunas zonas del Duero y de La Mancha. Como conclusión propone que se deben realizar prospecciones más detalladas y que es preciso disponer de valores horarios de velocidad. El resto de la publicación se refiere a la descripción de los distintos sistemas de pequeña y gran potencia, y finaliza con una estimación de la potencia eléctrica de origen eólico que podría instalarse en España.

En 1979 el Ministerio de Industria y Energía, a través del Centro de Estudios de la Energía, pone en marcha un Programa de Investigación y Desarrollo para el aprovechamiento de la energía eólica y su conversión en electricidad. El primer paso que se da tiene su fruto en el diseño y fabricación de una máquina experimental de $100 \mathrm{~kW}$ a una velocidad media de $12 \mathrm{~m} / \mathrm{seg}$. Para su emplazamiento se hace en el Instituto de Técnica Aeroespacial un estudio previo de las curvas de potencial eólico en España. Se escoge Tarifa, en la provincia de Cádiz, por ser el área que presenta un mayor número de horas de viento al año con un régimen de gran uniformidad y una densidad de potencia de más de 55 vatios $/ \mathrm{m}^{2}$ de media anual.

Los trabajos realizados desde 1981 a 1986 en el sector de la energía eólica en España consiguen desarrollos e instalaciones de máquinas de pequeña y mediana potencia que permiten dar un contenido tecnológico básico a la naciente industria del sector. En 1981 el Centro para el Desarrollo Tecnológico e Industrial, dependiente del Ministerio de Industria, Comercio y Turismo, convoca dos concursos para el desarrollo de prototipos de aerogeneradores de 5 a $10 \mathrm{~kW}$. Se construyen de esta forma las primeras máquinas nacionales de pequeña potencia. Por otro lado, el Programa Energético, fruto del convenio de la patronal eléctrica UNESA con el Instituto Nacional de Industria (P.E.U.I.), posibilita el desarrollo de otro modelo de aerogenerador de $24 \mathrm{~kW}$; instalado en Alfabia (Mallorca) va a servir como base del desarrollo de aerogeneradores de mayor potencia. En este contexto se construye en 1984 en El Ampurdán (Gerona) el primer parque eólico conectado a la red con cinco aerogeneradores de $24 \mathrm{~kW}$. La instalación se realiza mediante colaboración entre la Generalitat de Catalunya, la empresa eléctrica Empresa Nacional Hidroeléctrica Española Ribagorzana y el PEUI (Instituto para la Diversificación y Ahorro de la Energía, 1996).

Desde mediados de los años ochenta la tecnología eólica ha experimentado un enorme progreso en España. Se ha evolucionado desde máquinas de potencia unitaria de decenas de kilovatios, simples y con frecuencia de escasa fiabilidad, a aerogeneradores con una potencia de $2.000 \mathrm{~kW}$.

La tecnología ha permitido el desarrollo de máquinas de eje horizontal, tripalas, con alta calidad de suministro eléctrico, bajo mantenimiento y vida operativa que supera los veinte años. El diseño básico de los aerogeneradores que se fabrican en la actualidad admite modificaciones de altura de la torre y del diámetro de rotor, con el fin de optimizar el rendimiento energético de un emplazamiento con recursos eólicos determinados.

Entre las mejoras para captar la energía del viento, en los últimos años se han introducido novedades como torres más altas, rotores más potentes, palas más resistentes y de mayor envergadura, mejoras en la electrónica y mejor uso de los materiales compuestos. La mejora más impresionante ha sido el aumento en tamaño y rendimiento de los aerogeneradores. En el futuro se fabricarán turbinas incluso más grandes destinadas a parques eólicos marinos, que constituyen un mercado emergente de gran interés, debido a que además de causar un menor impacto paisajístico, ofrecen la ventaja de vientos más fuertes 
y predecibles que en tierra firme, aunque los costes de construcción son mayores (Instituto para la Diversificación y Ahorro de la Energía, 2003). En la Conferencia Eólica Europea celebrada en Madrid durante los días 16 a 19 de julio de 2003, J.L. García Ortega, responsable de energía de Greenpeace España ha presentado el informe Viento en popa, donde se plantea que un país que ha sabido empezar a sacarle partido al viento terrestre, debe aprovechar el viento marino. Parte de un concepto de utilidad de la eólica marina como fuente de energía para distintos vectores energéticos destinados a necesidades diferentes: conversión industrial para inyección de corriente alterna en la red, electrólisis para producción de hidrógeno, y desalación de agua marina. Lo primero y fundamental que contempla el plan es determinar los criterios para seleccionar los emplazamientos, para definir las zonas adecuadas, y de este modo poder analizar el potencial energético disponible. En la actualidad es posible ubicar aerogeneradores anclados a una profundidad de hasta treinta metros, espacio ocupado por una franja costera más o menos amplia, pero muy sensible a diferentes aspectos relacionados con los ecosistemas marinos, con la actividad de barcos pesqueros y mercantes, y también con la visibilidad de las instalaciones desde la costa. Por ello hay que comenzar estableciendo unos criterios de selección de emplazamientos que tengan en cuenta adecuadamente criterios biológicos, de tráfico marino y aspectos turísticos, entre otros. Viento en popa adelanta los criterios de especial interés para Greenpeace, ya que el de mayor velocidad del viento no debe ser el único a la hora de definir posibles emplazamientos de parques eólicos. Aparte del factor energético hay otros que también se relacionan con el medio ambiente y los aspectos sociales: profundidad, fondos, presencia de especies sensibles o amenazadas, interacción con otras actividades (caladeros utilizados por pesca artesanal) y tráfico marítimo.

Viento en popa realiza una primera estimación de potencial eólico marino en España, que sintetizado comprende: muelles y diques portuarios existentes (500 MW), País Vasco, Cantabria, Asturias y Galicia (500 MW), Golfo de Cádiz (10.000 MW), Costa mediterránea (7.500-15.000 MW) e Islas Canarias (varios cientos de MW). Serán precisos análisis más detallados con sus correspondiente mediciones para determinar las zonas con más precisión.

Viento en popa es sólo una primera reflexión que expone el punto de vista de Greenpeace sobre esta tecnología, y que tiene como objetivo mostrar la necesidad del estudio especializado, de la información y del consenso social. Serán precisas unas etapas que incluyan los siguientes aspectos: estudio del potencial eólico a diferentes profundidades a lo largo de todas las costas españolas, establecimiento de normas ambientales para futuros parques eólicos marinos, valorización ambiental de los posibles emplazamientos, criterios técnico-económicos de construcción de parques, esquemas de retorno ambiental, retorno y participación social, programa de desarrollo eólico marino, parques eólicos marinos de demostración, y ensayos de producción de hidrógeno con electricidad eólica. Un plan de esta envergadura sólo será posible si existe la voluntad política para su desarrollo (García, 2003).

El septiembre de 2003 se presenta el proyecto del primer parque eólico español, localizado en la costa de Cádiz, a unos $18 \mathrm{Km}$. del cabo de Trafalgar, entre Barbate y Conil, y con una potencia prevista de $1.000 \mathrm{MW}$. El proyecto, que incluye la instalación de jaulas de acuicultura en la base de los aerogeneradores, ha sido bien acogido por las organizaciones ecologistas, pero no por las autoridades locales y los pescadores. Los representantes de la flota de Barbate y Conil han mostrado un rechazo radical al proyecto por entender que pone en riesgo la pesca artesanal y afectaría también a las rutas migratorias de los atunes que pasan por el Estrecho. El impacto visual y la resistencia de los pescadores son dos de las razones expuestas por el alcalde de Barbate para rechazar el 
proyecto. J. L. García Ortega (Greenpeace) manifiesta que «estaremos a favor del proyecto si se garantiza resolver los impactos visuales, no genera perturbaciones en las rutas migratorias de aves en el Estrecho, no afecta a los caladeros de pesca litoral, y asegura la evacuación de la electricidad generada sin problemas» (Bolaños, 2003).

\section{Evolución de la potencia eólica instalada}

En España, al margen de los molinos que aprovechaban la energía eólica con fines mecánicos desde inicios de la Edad Media, y que conocieron un gran auge a finales del siglo XIX y primer tercio del siglo XX, la utilización de aerogeneradores para la producción de electricidad se inició en 1978 con la instalación de un prototipo de $100 \mathrm{~kW}$ en Tarifa (Cádiz).

Desde entonces, el esfuerzo realizado por el sector eléctrico en proyectos de investigación y demostración ha dado lugar a un notable desarrollo del aprovechamiento de la energía eólica para la producción de electricidad. España se sitúa entre los países que más han potenciado esta forma de energía, hasta el punto que en la actualidad ocupa el tercer puesto a nivel mundial, sólo superado por Alemania y Estados Unidos.

En España el crecimiento de la potencia y producción de energía eólica durante los años noventa ha sido espectacular. En el período comprendido entre los años 1990 y 2002 se ha pasado de una potencia instalada de 2 a $4.526 \mathrm{MW}$, y de una producción de $186.000 \mathrm{~kW}$ a 9.594 gigavatios (GWh). Esta aportación, en 2002, supone el $4 \%$ de la electricidad demandada en la España Peninsular y el $5 \%$ en las Islas Canarias (cuadro 1).

Cuadro 1

EVOLUCIÓN DE LA POTENCIA EÓLICA INSTALADA, PRODUCCIÓN Y APORTACIÓN A LA DEMANDA DE ELECTRICIDAD. 1990-2002

\begin{tabular}{|c|r|r|r|r|}
\hline Año & \multicolumn{1}{|c|}{$\begin{array}{c}\text { Potencia } \\
\text { MW }\end{array}$} & $\begin{array}{l}\text { Producción } \\
\text { GWh }\end{array}$ & $\begin{array}{c}\text { \% Demanda } \\
\text { Peninsular }\end{array}$ & $\begin{array}{c}\text { \% Demanda } \\
\text { Canarias }\end{array}$ \\
\hline 1990 & 2 & 2 & 0 & 0 \\
\hline 1991 & 7 & 3 & 0 & 0 \\
\hline 1992 & 33 & 17 & 0 & 0 \\
\hline 1993 & 34 & 85 & 0 & 0 \\
\hline 1994 & 39 & 78 & 0 & 0 \\
\hline 1995 & 89 & 181 & 0 & 0 \\
\hline 1996 & 181 & 374 & 0 & 1 \\
\hline 1997 & 361 & 697 & 0 & 2 \\
\hline 1998 & 838 & 1.354 & 1 & 2 \\
\hline 1999 & 1.521 & 2.696 & 1 & 4 \\
\hline 2000 & 2.202 & 4.699 & 2 & 4 \\
\hline 2001 & 2.844 & 6.931 & 3 & 5 \\
\hline 2002 & 4.526 & 9.594 & 4 & 5 \\
\hline
\end{tabular}

Fuente: Comisión Nacional de Energía y Red Eléctrica de España. 
Este extraordinario desarrollo de la energía eólica se explica por tres razones: por una parte, la disponibilidad de amplias zonas con potencial eólico estimable (en función de las velocidades, frecuencias y direcciones de los vientos), por otra por la legislación a nivel nacional, con políticas de apoyo decidido al recurso eólico, y por último, y quizás el estímulo más importante, por las políticas regionales de desarrollo impulsadas por las Comunidades Autónomas, como es el caso de Galicia, Navarra o Aragón que han recibido un impulso crucial desde los gobiernos autonómicos.

Por tanto, el desarrollo de la energía eólica no ha afectado de igual modo a todas las Comunidades Autónomas (cuadro 2). Durante el período comprendido entre los años 1998 y 2002 se han producido dos hechos reseñables. Por un lado, la incorporación de cuatro regiones a la producción de este tipo de electricidad: Comunidad Valenciana, País Vasco, La Rioja y Asturias. Por otra parte, se ha dado un crecimiento espectacular en la mayoría de las que ya contaban con parques eólicos en 1998. Llama la atención los casos de Castilla-La Mancha, que en 1998 aún no ha puesto en funcionamiento parques eólicos, y Castilla y León, que apenas dispone de 29 MW, en cambio en 2002 cada región cuenta con una potencia instalada próxima a los $600 \mathrm{MW}$. Galicia ha sextuplicado la capacidad en este breve período de tiempo, lo que le ha permitido continuar manteniendo un papel hegemónico entre las regiones que producen electricidad de origen eólico en España. Parecido comportamiento han tenido las comunidades del Valle del Ebro. Navarra, la región con más potencia en 1998, ha triplicado el volumen de la misma, y por tanto continúa siendo un referente dentro del conjunto de España; en Aragón, la construcción de parques eólicos ha sido tan intensa que ha dado lugar a que la potencia contabilizada en 2002 sea seis veces superior a la de 1998; reseñable también es el caso de La Rioja, que pese a su tardía incorporación a la producción de energía eólica, pasa de 24 a 204 MW de 2000 a 2002.

Cuadro 2

POTENCIA EÓLICA INSTALADA EN LAS COMUNIDADES AUTÓNOMAS. 1998-2002. (MW).

\begin{tabular}{|l|r|r|r|r|r|}
\hline & $\mathbf{1 9 9 8}$ & $\mathbf{1 9 9 9}$ & $\mathbf{2 0 0 0}$ & $\mathbf{2 0 0 1}$ & $\mathbf{2 0 0 2}$ \\
\hline GALICIA & 217 & 461 & 639 & 685 & 1.077 \\
\hline ARAGÓN & 135 & 209 & 212 & 412 & 893 \\
\hline NAVARRA & 237 & 336 & 457 & 568 & 686 \\
\hline CASTILLA-LA MANCHA & & 112 & 298 & 443 & 610 \\
\hline CASTILLA Y LEÓN & 29 & 129 & 228 & 299 & 571 \\
\hline LA RIOJA & & & 24 & 74 & 204 \\
\hline ANDALUCÍA & 112 & 124 & 143 & 150 & 166 \\
\hline CANARIAS & 68 & 82 & 104 & 117 & 127 \\
\hline CATALUÑA & 35 & 60 & 60 & 72 & 75 \\
\hline ASTURIAS & & & & 24 & 74 \\
\hline PAÍS VASCO & & & 24 & 24 & 27 \\
\hline MURCIA & 5 & 5 & 10 & 13 & 13 \\
\hline COMUNIDAD VALENCIANA & & 3 & 3 & 3 & 3 \\
\hline TOTAL & 838 & 1.521 & 2.202 & 2.884 & 4.526 \\
\hline
\end{tabular}

Fuente: Comisión Nacional de Energía y Red Eléctrica de España. 
La política de fomento llevada por la Unión Europea supone condiciones económicas y administrativas especiales para las energías renovables. La potencia eólica instalada en el conjunto de países que la integran ha pasado de 2.529 MW en 1995 a 20.284 MW en 2002, y se mantiene el ritmo de crecimiento. Como se expone en el cuadro 3 cada país presenta una tendencia distinta, aunque unos pocos acumulan la mayor parte de la capacidad instalada en la Unión Europea.

Cuadro 3

POTENCIA EÓLICA INSTALADA EN LOS PAÍSES DE LA UNIÓN EUROPEA (MW)

\begin{tabular}{|l|r|r|r|r|}
\hline & $\mathbf{2 0 0 1}$ & $\mathbf{2 0 0 2}$ & JUN-03 & \multicolumn{1}{c|}{ TOTAL } \\
\hline ALEMANIA & 8.754 & 10.650 & 12.836 & 52,13 \\
\hline ESPANA & $\mathbf{3 . 3 5 0}$ & $\mathbf{4 . 0 7 9}$ & $\mathbf{5 . 0 6 0}$ & $\mathbf{2 0 , 5 5}$ \\
\hline DINAMARCA & 2.417 & 2.515 & 2.916 & 11,84 \\
\hline PAISES BAJOS & 493 & 563 & 803 & 3,26 \\
\hline ITALIA & 697 & 755 & 800 & 3,25 \\
\hline REINO UNIDO & 474 & 530 & 586 & 2,38 \\
\hline SUECIA & 290 & 304 & 364 & 1,48 \\
\hline GRECIA & 272 & 276 & 354 & 1,44 \\
\hline FRANCIA & 78 & 131 & 220 & 0,89 \\
\hline AUSTRIA & 94 & 100 & 219 & 0,89 \\
\hline PORTUGAL & 125 & 171 & 217 & 0,88 \\
\hline IRLANDA & 125 & 125 & 137 & 0,56 \\
\hline BÉLGICA & 31 & 31 & 56 & 0,23 \\
\hline FINLANDIA & 39 & 39 & 41 & 0,17 \\
\hline LUXEMBURGO & 15 & 15 & 16 & 0,06 \\
\hline TOTAL & 17.254 & 20.284 & 24.625 & 100 \\
\hline
\end{tabular}

Fuente: The European Wind Energy Association.

\section{Localización de los parques eólicos}

Las regiones con presencia de vientos frecuentes son las primeras que han instalado parques eólicos, localizados dentro de su ámbito territorial en los lugares en los que las mediciones han permitido conocer la viabilidad de la instalación de aerogeneradores. Todas ellas se encuentran dentro de las zonas de España relacionadas por Olcina (1994) y señaladas en la introducción de este artículo.

A 31 de diciembre de 2002, tal y como se expone en el cuadro 2, Galicia es la Comunidad Autónoma que dispone de mayor potencia eólica instalada, más de un millar de MW, que suponen el 23,8 \% del total nacional. El Gobierno Gallego con el Plan Eólico de Galicia pretende alcanzar en 2010 una capacidad de $4.000 \mathrm{MW}$, aproximadamente el 55 $\%$ de la demanda de electricidad prevista. Detrás se sitúan Aragón (19,7 \%) y Navarra 
$(15,2 \%)$. En Navarra ha sido y es fundamental el papel de la empresa Energía Hidroeléctrica de Navarra, que se ha convertido en uno de los promotores eólicos más importantes a nivel mundial. Castilla-La Mancha junto con Castilla y León cuentan con una cifra de MW en funcionamiento que les permite a cada una de estas regiones una aportación en torno al $13 \%$. En total estas cinco Comunidades acumulan el $85 \%$ de la potencia instalada en nuestro país al finalizar el año 2002.

La Rioja, Andalucía, Canarias, Cataluña y Asturias cuentan con una potencia que contribuye con un porcentaje que oscila entre 4,5\% de La Rioja al 1,6\% de Asturias.

Los parques eólicos tienen un carácter testimonial en el País Vasco, Región de Murcia y Comunidad Valenciana, y a finales de 2002 no se han instalado o puesto en funcionamiento en las comunidades de Cantabria, Extremadura, Madrid e Islas Baleares.

\section{La industria eólica en España}

El desarrollo inicial de la industria eólica se debe a pequeñas empresas que hacían sus propios diseños de aerogeneradores y compraban en el mercado los distintos componentes, para ensamblarlos primero en naves de montaje y posteriormente en talleres más especializados. Se fueron desarrollando así lazos de colaboración entre diseñadores y fabricantes de componentes, a la vez que se incrementaba el volumen de trabajo de éstos últimos, que también eran pequeñas empresas. Poco a poco algunos fabricantes fueron especializándose o creando algún taller con dedicación mayoritaria a la industria eólica. En España se ha ido configurando una industria de aerogeneradores cada vez más potente, que se mueve en un mercado internacional de creciente competitividad. Estas empresas han evolucionado al ritmo de la potencia instalada en energía eólica (Menéndez, 2001).

El sector industrial eólico español es líder a nivel mundial. Todas las compañías más importantes tienen plantas en nuestro país, y además ocupa el tercer puesto en el mundo por capacidad instalada. Existe un mercado exterior incipiente y con alto potencial que es un objetivo claro para las empresas españolas.

En el año 2000 este sector cuenta con 17.000 empleados, 5.000 directos y 12.000 indirectos, y está prevista la creación de más de 35.000 puestos de trabajo para 2010. El sector de energía eólica español es intensivo en I+D con una inversión muy superior a la media española. Su innovación está enfocada en los sectores denominados clave en el Plan Nacional de Investigación Científica, Desarrollo e Innovación Tecnológica del Ministerio de Ciencia y Tecnología.

Según la Plataforma Empresarial Eólica, más de 300 empresas participan actualmente en la industria eólica española. A continuación se relaciona el número total según sectores de actividad y las principales por orden alfabético:

a) Fabricación de componentes (140): ABB, Alstom Power, Apoyos Matálicos Olezti, Fiderblade, Flender-Winergy, Gamesa Eólica, Izar, Mita.Teknik, Siemsa, Soluciones Energéticas, Treólica y Xantrex.

b) Fabricación de equipos (15): Ecotècnia, Enercon, Gamesa Eólica, GE Wind, Izar Bonus, MADE, Neg Micon.

c) Promoción y explotación (140): Abo Wind, Aerogeneradores Canarios, Álabe, Cannon Power, Cesa, Desa-Nuon, Corporación Energía Hidroeléctrica de Navarra, Endesa, Enerfín, Energi, Eólica Navarra, Eurovento, Eyra, Gamesa Energía, Gecalsa, H-10 Grupo de participaciones, Iberdrola, NUON, Palencia Energía Eólica, Preneal, Sinae, Sistemas de Energías Regenerativas, Sociedad Eólica de Andalucía. 
d) Construcción y servicios (50): 360 Corporate Finance, Abengoa, Acciona, ACS, Alatec, Bancsabadell, Barlovento, Beas de Ingeniería, Cobra, Dragados, Elecnor, Ferrovial, Iberinco, Infopower, Isastur, La Caixa, Marsh, San Martín, Semi, Unibrok.

e) Transporte y distribución (6): Endesa, Hidrocantábrico, Iberdrola. Nueva Viesgo, Red Eléctrica de España y Unión Fenosa.

El fuerte desarrollo de la energía eólica a lo largo de la década de los noventa, según E. Menéndez (2001), ha dado lugar a un esquema industrial en el que aparecen dos tipos de fabricantes de aerogeneradores:

- Empresas con diseños propios, que proceden del primer período de desarrollo autóctono y han avanzado a partir de su propio esfuerzo tecnológico, es el caso de Ecotècnia y MADE.

- Empresas con origen tecnológico en otros países europeos que tienen diferentes esquemas de implantación en España. Entre ellas destacan Gamesa Eólica, perteneciente a un grupo industrial autóctono, que se desarrolla a partir de tecnología danesa de Vestas, conectada con el grupo energético Iberdrola. Otras empresas significativas son Izar-Bonus, Neg-Micom y Nordex.

Gamesa, MADE y Ecotècnia, han suministrado el $80 \%$ de la potencia instalada en nuestro país. Sólo Gamesa acapara más del 54 \% del mercado (cuadro 4). Dada la envergadura de estas tres industrias parece oportuno hacer una breve reseña de las mismas, hecho que ha sido posible con la información obtenida en Internet (www.gamesa.es / www. made.es / www.ecotecnia.ecom).

Cuadro 4

DISTRIBUCIÓN POR FABRICANTES DE LA POTENCIA INSTALADA EN PARQUES EÓLICOS DE ESPAÑA A 31 DE DICIEMBRE DE 2002

\begin{tabular}{|l|c|}
\hline GAMESA EÓLICA & $54,05 \%$ \\
\hline MADE TECNOLOGÍAS RENOVABLES & $13,09 \%$ \\
\hline ECOTĖCNIA & $10,27 \%$ \\
\hline NEG MICON SAU & $8,47 \%$ \\
\hline GENERAL ELECTRIC WIND & $6,15 \%$ \\
\hline IZAR-BONUS & $3,58 \%$ \\
\hline DESARROLLOS EÓLICOS & $1,52 \%$ \\
\hline LAGERWEY & $0,76 \%$ \\
\hline NORDEX & $0,70 \%$ \\
\hline KENETECH & $0,60 \%$ \\
\hline ABENGOA W.P. & $0,40 \%$ \\
\hline ENERCON & $0,35 \%$ \\
\hline AEROGENERADORES CANARIOS & $0,07 \%$ \\
\hline
\end{tabular}

Fuente: Revista Infopower, n 51, nov./dic. 2002. 
Gamesa Eólica se constituye en 1994 y en la actualidad es uno de los cinco principales fabricantes mundiales de aerogeneradores. En 2000 alcanzó el segundo puesto mundial con una cuota de mercado del 13,9 \%. En España es líder en el sector de fabricación, venta e instalación de turbinas eólicas, con una cuota de mercado acumulada del $54 \%$ a finales de 2002.

Esta sociedad cuenta con amplia capacidad propia de diseño y desarrollo tecnológico de aerogeneradores. Ofrece una amplia gama de productos con potencias comprendidas entre los $660 \mathrm{~kW}$ y los $2 \mathrm{MW}$. La comercialización, instalación y mantenimiento de estos equipos los realiza a escala mundial. Cuenta con delegaciones comerciales en Francia, Italia, Grecia, Portugal y Brasil, y con próxima apertura en Estados Unidos y Alemania. Esta red comercial se completa con acuerdos de colaboración con empresas de primer nivel en países como Australia y Japón. Su departamento de I+D está formado por más de 150 personas dedicadas a diseñar y desarrollar nuevos aerogeneradores; identificar, especificar y desarrollar proyectos de innovación tecnológica de productos y procesos productivos; y a proporcionar el soporte técnico necesario a las diferentes actividades de fabricación, instalación y servicio que lleva a cabo la empresa. A esta capacidad de diseño tecnológico propia se une una capacidad productiva integral. Gamesa Eólica diseña y fabrica sus propias palas, moldes para la fabricación de palas, raíces de pala, multiplicadoras y torres, además realiza el ensamblaje del aerogenerador. Cuenta con 12 centros de producción en España. Esta amplia capacidad industrial le permite controlar íntegramente el proceso de producción, desde el diseño a la fabricación de los distintos componentes críticos del aerogenerador.

Gamesa Eólica es capaz de ofrecer el suministro completo del parque eólico en su modalidad «llave en mano». Realiza el suministro, montaje y puesta en marcha de los aerogeneradores y equipos asociados, mientras que Gamesa Energía Servicios (sociedad participada en su totalidad por Gamesa) realiza la obra civil, conexiones eléctricas, subestación y líneas de alta tensión. Esta compañía, entre los años 1997 y 2001, ha pasado de una facturación de 66,45 a 470,34 millones de euros, y una plantilla de 214 a 1.114 empleados.

MADE Tecnologías Renovables. inició su trayectoria en 1940 como fabricante de componentes para la electrificación. En la actualidad cuenta con una experiencia de veinte años en el campo de las energías renovables. Esta empresa forma parte del grupo Endesa, encontrándose su accionariado en manos de Endesa Diversificación en su totalidad. Su actividad se centra hoy en día en el diseño y fabricación de aerogeneradores y colectores solares. Cuenta con dos centros de trabajo: la fábrica de Medina del Campo (Valladolid), con una plantilla de 70 personas, y las oficinas de Madrid donde se concentra gran parte del desarrollo eólico, en sus facetas comercial y de investigación y desarrollo. Dispone de cuatro aerogeneradores con potencias comprendidas entre $660 \mathrm{~kW}$ y $2 \mathrm{MW}$.

La ejecución de parques eólicos en la modalidad «llave en mano» es uno de los productos/servicios que MADE puede facilitar a sus clientes, con la intención de simplificar y optimizar la gestión que éstos llevan a cabo en su intención de construir una instalación de este tipo. La empresa se responsabiliza de toda la gestión de compras, planificación, construcción, montaje y puesta en marcha de la totalidad de elementos que integran la instalación.

Ecotècnia Societat Cooperativa se funda en 1981. Desde el principio su actividad ha estado exclusivamente orientada al desarrollo de aplicaciones de las energías renovables. Desde abril de 1999 Ecotècnia está integrada en Mondragón Corporación Cooperativa. En la actualidad se centra preferentemente en el diseño y fabricación de aerogeneradores de elevada potencia y en la promoción, construcción y mantenimiento de parques eólicos. Sus 
centros de producción se localizan en Buñuel (Navarra) y As Somozas (La Coruña) para la fabricación eólica y cuenta con una planta en Zamora dedicada a la fabricación de torres para aerogeneradores. La gama de aerogeneradores comprende modelos con una potencia que va desde 640 a $1.670 \mathrm{~kW}$. En 2001 cuenta con una plantilla de 260 empleados y una facturación que asciende a 6,32 millones de euros.

En España los agentes regionales fomentan el desarrollo industrial en sus áreas de influencia a partir de la idea de que se utilizan recursos energéticos autóctonos y las implantaciones de parques ocupan terrenos, también propios, y agreden (si es el caso) a su entorno. En este contexto, las industrias de fabricación de componentes se están desarrollando en nuestro país con una cierta tendencia a ubicarse en las mismas regiones en las que se instalan los parques eólicos. Esto tiene un aspecto positivo dado que en la mayoría de estas regiones el entramado industrial es de baja densidad, con lo cual se crea empleo en zonas que lo necesitan. Galicia, Castilla-La Mancha, Castilla y León, Andalucía y Aragón son un ejemplo de este planteamiento (Menéndez, 2001). Galicia ha sido la región que más ha insistido en este aspecto, ya que se ha solicitado a los promotores de parque eólicos un plan industrial paralelo al desarrollo eólico. De hecho, el Plan Eólico de Galicia, que tiene como objetivo la instalación de unos 4.000 MW de potencia, contempla además la creación de 2.100 empleos, distribuidos en fábricas de componentes (600), industria auxiliar (400), construcción de parques y líneas eléctricas (500), servicios de ingeniería y promoción (400), y mantenimiento de parques (200).

\section{La energía eólica y el medio ambiente}

La energía eólica es una opción limpia, pero la instalación de un parque eólico genera un impacto ambiental que es preciso evaluar de acuerdo con las condiciones del entorno, tanto físico como social, con el fin de introducir las medidas correctoras oportunas. Existe un amplio consenso en nuestra sociedad sobre la coexistencia de las instalaciones eólicas y los entornos en los que se asientan, si bien se producen determinados impactos derivados del aprovechamiento de la energía eólica que no se deben obviar.

En la publicación La energía eléctrica y el medio ambiente, editada por UNESA (Asociación Española de la Industria Eléctrica) en 2001, se relacionan los impactos de la energía eólica sobre el medio ambiente:

a) Sobre el entorno, los aerogeneradores eléctricos no producen emisiones contaminantes (atmosféricas, residuos, vertidos líquidos) y por tanto no contribuyen al efecto invernadero. No obstante, tienen consecuencias medioambientales que dependen fundamentalmente del emplazamiento elegido para su instalación, de su tamaño y de su distancia respecto a zonas de concentración de población. Una instalación eólica de gran tamaño produce alteraciones en el medio físico -impacto visual, sobre las aves, ruido y erosión- y en el medio socioeconómico. Las primeras afectan sólo a la superficie ocupada y zonas colindantes. El impacto sobre las aves es pequeño y de tipo fundamentalmente indirecto: posibles colisiones con líneas aéreas y con torres soportes de cables. De hecho, la colisión no suele producirse contra los aerogeneradores, porque las aves se acostumbran rápidamente a su existencia y a su movimiento, e incluso las migratorias desvían su trayectoria cuando el parque eólico se encuentra en la dirección de su vuelo.

b) Los elementos característicos de una instalación eólica que producen impacto visual son los aerogeneradores, la caseta, las líneas eléctricas y los accesos a la instalación. Para evitarlo en la medida de lo posible, suelen emplearse colores adecuados, una 
inserción apropiada de las instalaciones en la orografía del lugar y una cuidada distribución de los aerogeneradores. En relación con los accesos, se evita al máximo el movimiento de tierras, recuperando inmediatamente la cubierta vegetal afectada y autorizando el paso únicamente al personal de las instalaciones. Por otro lado, el impacto visual de estas instalaciones depende de criterios subjetivos: mientras que un parque de unos pocos aerogeneradores puede llegar a ser incluso atractivo, una gran concentración de máquinas plantea problemas mayores.

c) En cuanto al efecto sonoro, un aerogenerador produce un ruido similar al de cualquier otro equipamiento industrial de la misma potencia, con la diferencia de que, mientras los equipos convencionales se encuentran normalmente encerrados en edificios o compartimentos diseñados para minimizar su nivel sonoro, los aerogeneradores deben trabajar al aire libre y cuentan con un elemento transmisor del sonido, que es el propio viento.

d) En relación con los impactos por erosión, la mayor incidencia se produce por el movimiento de tierras en la preparación de los accesos al parque eólico. Esta incidencia se puede reducir mediante estudios de trazado y perfiles transversales del camino, así como análisis de impacto sobre la vegetación de las vaguadas y cursos de agua.

La incidencia de los aerogeneradores sobre las aves no es tan simple como se ha descrito. Una gran parte de las instalaciones eólicas se ubican siguiendo las líneas de cumbres, que son empleadas por las aves para remontar su vuelo, aprovechando las corrientes termodinámicas que generan. Cuando bajo la instalación no se produce un descenso suave, en forma de talud, sino que se abre un cantil abrupto, la instalación se vuelve barrera infranqueable e invisible a las aves que, como mucho, identifican los mástiles pero no las palas de los aerogeneradores. Todo esto depende también mucho de las especies. En el caso de las migratorias, que su recorrido nunca es exactamente el mismo, unos cientos de metros de alejamiento de su ruta les puede salvar la vida, aunque ello no quita que al año siguiente, en su desplazamiento migratorio, puedan colisionar allí donde no lo hicieron el anterior. Una situación distinta se da con las aves sedentarias, que nidifican en las sierras donde se instalan los parques eólicos y deben atravesarlos varias veces al día. Entre las aves más afectadas, una gran parte de ellas protegidas, están las esteparias de gran porte (avutardas y sisones), rapaces rupícolas (águila real, águila perdicera y halcón peregrino), las planeadoras (cigüeña, milano, águila calzada, buitres en general) y todas aquellas de actividad vespertina o nocturna, para las que las palas son indetectables. A esta conclusión se ha llegado gracias a la realización de informes sobre la mortandad de aves en parques eólicos. Un análisis crítico de cuatro de estos informes está disponible en Internet: $w w w . i b e r i c a 2000 . o r g / E s / a r t i c u l o . a s p ? I d=1136$. Dos de estos analizan ámbitos territoriales españoles con destacada presencia de parques eólicos: Incidencia de las plantas de aerogeneradores sobre la avifauna en la comarca del Campo de Gibraltar, realizado por Luis Barrios, de la Sociedad Española de Ornitología en junio de 1995, por encargo de la Agencia de Medio Ambiente de la Junta de Andalucía, y cuyas conclusiones están publicadas en: www.iberica2000.org/Es/Articulo.asp?Id=675. Jesús $\mathrm{M}^{\mathrm{a}}$ Lekuona redacta en abril de 2001, para la Dirección General de Medio Ambiente del Gobierno de Navarra, el documento Uso del espacio por la avifauna y control de la mortalidad de aves y murciélagos en los parques eólicos de Navarra durante un ciclo anual, y cuya consulta es posible en www.iberica2000.org/documents/EOLICA/LEKUONA_REPORT.pdf. Además de las abundantes conclusiones que aporta, incluye las recomendaciones a tener en cuenta con el fin de disminuir la mortalidad real de la avifauna y las numerosas situaciones de riesgo que se han detectado durante todo el periodo de estudio, junto con la necesidad de 
plantear un protocolo de trabajo que afecte a las nuevas instalaciones eólicas que se vayan a instalar en la Comunidad Foral de Navarra.

\section{La energía eólica en la Política de Fomento de las Energías Renovables}

Las razones para el fomento desde los poderes públicos de las energías renovables tienen su origen en la problemática provocada por las emisiones contaminantes en el Planeta que puede ser grave a medio plazo. De ahí que los países desarrollados deben afrontar políticas de ahorro y eficiencia energética, y de fuerte impulso de las energías renovables, así como dinámicas de transferencia tecnológica a los países menos desarrollados, de manera que su crecimiento económico se sustente en modelos energéticos menos agresivos para el ecosistema (Fernández de Salamanca, 2001).

El impulso más significativo y la concienciación general sobre la problemática de las emisiones contaminantes comienza con la celebración de la Cumbre de la Tierra en la reunión de 106 países en Río de Janeiro en el año 1992. Uno de los logros más llamativos de la Cumbre corresponde a la Convención Marco sobre Cambio Climático, que compromete a la comunidad internacional con un objetivo ambicioso pero crucial: llevar la estabilidad a la atmósfera global.

La Cumbre de Kioto sobre el Clima, celebrada en diciembre de 1997, ha precisado que Europa deberá reducir sus emisiones de gases de efecto invernadero un $8 \%$ entre los años 2008 y 2012, mientras que Estados Unidos lo hará en un 7\% y Japón un 6\%.

Por ello resulta evidente que tanto Europa como el conjunto de los países desarrollados económicamente, como principales responsables de la contaminación que padece el Planeta, tendrán que hacer un esfuerzo importante en la implantación de energías limpias, todo ello sin olvidar los programas de ahorro y eficiencia energética.

El Libro Blanco de la Comisión de las Comunidades Europeas Energía para el Futuro: Fuentes de Energía Renovables propone una campaña de despegue real de las energías renovables para obtener una penetración a gran escala y avanzar hacia el objetivo ambicioso, pero realista, de incrementar la contribución de las fuentes de energía renovable en el consumo bruto de energía interior de la Unión Europea hasta el 12\% antes del año 2010. Este objetivo global implica que los Estados miembros tienen que fomentar el aumento de las mismas de acuerdo con su propio potencial. En el Libro Blanco de la Comisión de las Comunidades Europeas se enumeran los motivos por los que es necesario fomentar activamente las energías renovables:

- Protección del medio ambiente y reducción de las emisiones de gases contaminantes causantes del efecto invernadero (sobre todo $\mathrm{CO}_{2}$ ).

- Reducción de la creciente dependencia exterior de importaciones de energía.

- Fomento del desarrollo regional y creación de puestos de trabajo.

- Exportación de la industria europea al mercado mundial.

- Aceptación social de un uso más intensivo de las energías renovables.

El desarrollo tecnológico y la utilización progresiva de las energías renovables han sido apoyados desde la Administración Española mediante la elaboración de Planes Nacionales con objetivos específicos para cada una de las áreas.

En 1986 y 1989 se realizan los primeros Planes de Energías Renovables destinados sobre todo a proyectos de ampliación y demostración. Para el sector eólico estos planes suponen un gran avance en el desarrollo de la tecnología nacional, un mayor conocimiento de las condiciones del viento en las distintas áreas de implantación y la adquisición de 
experiencia en la problemática de la explotación y mantenimiento (Instituto para la Diversificación y Ahorro de la Energía, 1996).

En 1991 se aprueba un nuevo Plan Energético Nacional que incluye el Plan de Ahorro y Eficiencia Energética, y que, entre otros objetivos, contempla incrementar la producción con energías renovables en 1,1 millones de toneladas equivalentes de petróleo. Cualitativamente este Plan, en el área eólica, hace hincapie en el aprovechamiento de las áreas geográficas nacionales con alto potencial, con la implantación de parques eólicos conectados a la red de distribución eléctrica. También se considera prioritario el desarrollo tecnológico y la fabricación de aerogeneradores españoles.

El Plan de Fomento de Energía Renovables, aprobado por el Gobierno en diciembre de 1999, recoge los principales elementos y orientaciones que pueden considerarse relevantes en la articulación de una estrategia para que el crecimiento de cada una de las áreas de energía renovables pueda cubrir, en su conjunto, cuando menos, el $12 \%$ del consumo de energía primaria en el año 2010.

Este Plan se elabora como respuesta al compromiso que emana de la Ley 54/1997, de 27 de noviembre, del Sector Eléctrico, y que define el objetivo de desarrollo a alcanzar por las energías renovables. Esta Ley, en su artículo 27, define el «Régimen Especial de la Producción Eléctrica». En el punto 1 se señala que la actividad de producción de energía eléctrica tendrá la consideración de producción en régimen especial en los siguientes casos, cuando se realice desde instalaciones cuya potencia no supere los $50 \mathrm{MW}$ :

a) Autoproductores que utilicen la cogeneración u otras formas de producción de electricidad asociadas a actividades no eléctricas siempre que supongan un alto rendimiento energético.

b) Cuando se utilice como energía primaria alguna de las energías renovables no consumibles, biomasa o cualquier tipo de biocarburante, siempre y cuando su titular no realice actividades de producción en el régimen ordinario.

c) Cuando se utilice como energía primaria residuos no renovables.

También tendrá la consideración de producción en régimen especial la producción de energía eléctrica desde instalaciones de tratamiento y reducción de los residuos de los sectores agrícola, ganadero y de los servicios, con una potencia instalada igual o inferior a $25 \mathrm{MW}$, cuando suponga un alto rendimiento energético.

La elaboración del Plan de Fomento de Energías Renovables se ha basado en estudios realizados para la evaluación del potencial eólico disponible, labor compleja que requiere la utilización de técnicas y aparatos de medición específicos a situar en zonas concretas y durante tiempos suficientemente dilatados. Por otra parte, el potencial neto técnicamente aprovechable, esto es, aquel que ha tenido en cuenta limitaciones técnicas, económicas, sociales o medioambientales, es mucho más sensible a la evolución del nivel tecnológico de los aerogeneradores, es decir no se trata de un valor estable.

El Documento del Plan presenta la situación en el año 1999 y las previsiones para los próximos años de la producción mediante energías renovables, el estado de las tecnologías de aprovechamiento de este tipo de recursos y las barreras que limitan la penetración de estas fuentes en un mercado cada vez más liberalizado. Teniendo en cuenta los distintos factores analizados, el Plan establece los objetivos eólicos que se esperan conseguir en el año 2010, siempre que se eliminen las barreras más importantes como la capacidad de evacuación eléctrica, el consenso social, la armonización medioambiental y el mantenimiento del marco eléctrico normativo actual sin variaciones sensibles en las tarifas. Las 
inversiones asociadas al Plan de Fomento de Energías Renovables serían del orden de 7,2 millones de euros (1,2 billones de pesetas).

La participación de la energía eólica en los objetivos de generación eléctrica previstos por el Plan representa un $71 \%$. en términos de potencia y un $53 \%$ en términos de energía. Esta evolución previsible de la energía eólica va a permitir cubrir un buen porcentaje de los objetivos de generación eléctrica contribuyendo de manera eficaz al objetivo global de duplicar la participación de las energías renovables en el consumo de energía primaria.

El Plan de Fomento de Energías Renovables establece unas previsiones en el ámbito eólico, por Comunidades Autónomas, que a la vista de lo comentado anteriormente, se esperan cumplir en el horizonte del año 2010, y que son las que se indican en el cuadro 5. Como se puede ver, en 2002 ya se ha conseguido en el conjunto de España el 55,6 \% del objetivo previsto, con importantes contrastes entre los distintos ámbitos territoriales.

Cuadro 5

PLAN DE FOMENTO DE LAS ENERGÍAS RENOVABLES EN ESPAÑA. OBJETIVOS EÓLICOS DE POTENCIA POR COMUNIDAD AUTÓNOMA. (MW)

\begin{tabular}{|l|r|r|r|}
\hline & $\begin{array}{r}\text { Potencia } \\
\text { Instalada } \\
\mathbf{2 0 0 2}\end{array}$ & $\begin{array}{r}\text { Objetivo } \\
\text { del Plan } \\
\mathbf{2 0 1 0}\end{array}$ & $\begin{array}{r}\text { \% Potencia } \\
\text { Instalada/ } \\
\text { Objetivo }\end{array}$ \\
\hline GALICIA & 1.077 & 2.268 & 47,49 \\
\hline ARAGÓN & 893 & 872 & 102,41 \\
\hline NAVARRA & 686 & 398 & 172,36 \\
\hline CASTILLA-LA MANCHA & 610 & 400 & 152,50 \\
\hline CASTILLA Y LEÓN & 571 & 834 & 68,47 \\
\hline LA RIOJA & 204 & 100 & 204,00 \\
\hline ANDALUCÍA & 166 & 985 & 16,85 \\
\hline CANARIAS & 127 & 170 & 74,71 \\
\hline CATALUNA & 75 & 405 & 18,52 \\
\hline ASTURIAS & 74 & 300 & 24,67 \\
\hline PAÍS VASCO & 27 & 200 & 13,50 \\
\hline MURCIA & 13 & 294 & 4,42 \\
\hline COMUNIDAD VALENCIANA & 3 & 290 & 1,03 \\
\hline CANTABRIA & 0 & 300 & 0,00 \\
\hline EXTREMADURA & 0 & 225 & 0,00 \\
\hline BALEARES & 0 & 49 & 0,00 \\
\hline MADRID & 0 & 50 & 0,00 \\
\hline TOTAL & 4.526 & 8.140 & 55,60 \\
\hline
\end{tabular}

Fuente: Red Eléctrica de España e Instituto para la Diversificación y Ahorro de la Energía.

En el documento Planificación de los sectores de Electricidad y Gas. Desarrollo de las redes de transporte 2002-2011, aprobado por el Gobierno en septiembre de 2002, las estimaciones de potencia de energía eólica superan las previstas por el Plan de Fomento de 
las Energías Renovables. La previsión de producción de electricidad con energía eólica en 2011 es de una potencia de 13.000 MW y una producción de $28.600 \mathrm{GWh}$.

Debido al gran desarrollo de la energía eólica desde la aprobación del Plan de Fomento de Energías Renovables, en este Documento, aprobado a finales de 2002, se recopila como la mayoría de las Comunidades Autónomas han solicitado a Red Eléctrica de España la realización de estudios a la red de transporte de 400 y $200 \mathrm{KW}$, para energía eléctrica de fuente eólica procedente de redes de tensiones inferiores.

La potencia eólica prevista por las Comunidades Autónomas peninsulares en el año 2011 suma 30.025 MW (cuadro 6). Red Eléctrica de España había recibido hasta marzo de 2002 solicitudes de acceso a la red de transporte de parques eólicos para una potencia instalada superior a $40.000 \mathrm{MW}$.

Cuadro 6

POTENCIA INSTALADA EÓLICA PREVISTA POR COMUNIDADES

AUTÓNOMAS PENINSULARES. AÑO 2011. (MW).

\begin{tabular}{|l|r|r|r|}
\hline & $\begin{array}{r}\text { Potencia } \\
\text { Prevista } \\
\mathbf{2 0 1 1}\end{array}$ & $\begin{array}{r}\text { Potencia } \\
\text { Instalada } \\
\mathbf{2 0 0 2}\end{array}$ & $\begin{array}{r}\text { \% Potencia } \\
\text { Instalada/ } \\
\text { Prevista }\end{array}$ \\
\hline GALICIA & 4.000 & 1.077 & 26,93 \\
\hline ARAGÓN & 3.200 & 893 & 27,91 \\
\hline NAVARRA & 1.536 & 686 & 44,66 \\
\hline CASTILLA-LA MANCHA & 4.452 & 610 & 13,70 \\
\hline CASTILLA Y LEÓN & 6.579 & 571 & 8,68 \\
\hline LA RIOJA & 665 & 204 & 30,68 \\
\hline ANDALUCÍA & 4.000 & 166 & 4,15 \\
\hline CATALUNA & 1.073 & 75 & 6,99 \\
\hline ASTURIAS & 500 & 74 & 14,80 \\
\hline PAÍS VASCO & 250 & 27 & 10,80 \\
\hline MURCIA & 600 & 13 & 2,17 \\
\hline COMUNIDAD VALENCIANA & 2.820 & 3 & 0,11 \\
\hline CANTABRIA & 300 & 0 & 0,00 \\
\hline EXTREMADURA & 0 & 0 & 0,00 \\
\hline MADRID & 50 & 0 & 0,00 \\
\hline TOTAL & $\mathbf{3 0 . 0 2 5}$ & 4.399 & 14,65 \\
\hline
\end{tabular}

Fuente: Ministerio de Economía y Red Eléctrica de España.

En cuanto a la política de la Unión Europea, el Parlamento y el Consejo de Europa aprobaron el 28 de febrero de 2000 por medio de la Decisión 646/2000 un programa plurianual, denominado Altener, destinado a fomentar las energías renovables en el seno de la Unión Europea. Se pretende así impulsar la inversión, mediante ayudas económicas a pequeñas y medianas empresas con el fin de aumentar la capacidad operativa de producción de energía a partir de fuentes renovables. 
Los objetivos del programa Altener se centran en limitar las emisiones de dióxido de carbono, aumentar el consumo interno bruto de energía en la Unión en 2010, disminuir la dependencia en las importaciones de energía, asegurando el abastecimiento y el fomento del empleo y fortaleciendo el potencial económico de las regiones remotas y periféricas. Todo ello mediante la consecución de dos objetivos específicos tales como contribuir a crear las condiciones jurídicas, socioeconómicas y administrativas necesarias para la aplicación del plan de acción comunitario sobre energías renovables, y en segundo lugar impulsar la inversión pública y privada en la producción y el consumo de energía derivada de fuentes renovables.

Para poner en práctica los mencionados objetivos, la Decisión 646/2000 prevé un sistema de financiación mediante el cual la Unión Europea sufragará:

- Los costes relativos a estudios que potencien las energías renovables, tales como la preparación de estrategias sectoriales y de mercado, elaboración de normas y sistemas de certificación, análisis comparativos o estudios de repercusión en el medio ambiente.

- Las acciones específicas que faciliten la penetración en el mercado de dichas fuentes de energía, prestando asesoramiento para la preparación y presentación de proyectos, así como para su aplicación.

- Las medidas de control, destinadas en general, a prestar apoyo a las iniciativas de aplicación del plan de acción, evaluando la repercusión y la relación coste-eficacia de las acciones y medidas adoptadas con arreglo al programa Altener.

\section{Conclusiones}

Desde mediados de los años noventa España ha apostado por la energía eólica y se ha convertido en una potencia mundial en el aprovechamiento de esta fuente energética. Ello ha sido posible gracias a la política de apoyo de las administraciones y al desarrollo espectacular de las industrias españolas que se dedican a este sector. Según la Plataforma empresarial eólica, el sector eólico invierte en investigación y desarrollo el $11 \%$ del valor añadido bruto de las empresas, porcentaje muy por encima de sectores considerados punteros como el electrónico, el químico y el informático, que se sitúan entre el $3 \%$ y $6 \%$.

La implantación de parques eólicos en áreas rurales con escaso desarrollo económico supone una gran fuente de ingresos para los municipios. Los ayuntamientos los obtienen de las licencias de obras, impuesto de actividades económicas y de la renta percibida cuando los aerogeneradores se instalan en terrenos de propiedad municipal. La cantidad percibida en concepto de alquiler del suelo se sitúa alrededor de 2.000 euros al año por cada aerogenerador. Los habitantes de estos territorios, además de alquilar sus tierras a las empresas con este fin, pueden encontrar empleo durante el tiempo que dura la construcción y que, dada la envergadura de los parques, se suele prolongar durante varios meses. El caso del municipio de La Muela, en la provincia de Zaragoza, es significativo ya que cuenta con 500 aerogeneradores, la mitad asentados en suelo de titularidad municipal.

Mientras que desde algunos sectores se cuestiona este modelo energético de desarrollo de las energías renovables, debido a las subvenciones que perciben, ecologistas, sindicatos y la patronal de este sector tienen una opinión distinta. Para Greenpeace la prima hay que entenderla como un incentivo, no como una subvención, es un reconocimiento a los beneficios ambientales. En vez de penalizar las energías contaminantes, las 
que parten de los combustibles fósiles, y no reflejan en su precio los considerados costes ambientales, lo que contaminan, se decide otro sistema: incentivar las limpias. Según la Asociación de Energías Renovables, con frecuencia se afirma que estas energías son muy caras para descalificarlas como opción energética, pero esta opinión no es cierta debido a que las energías renovables incorporan en su retribución todos los costes en que incurren, mientras que las energías convencionales han externalizado la mayor parte de sus costes. ¿Qué quiere decir esto? Que cuando el sistema eléctrico español paga un kWh renovable a una determinada cantidad, en la misma están recogidos todos los costes que ha originado su producción. Sin embargo, cuando el sistema paga un kWh convencional a una cantidad inferior — que lleva a la conclusión de que éstos son más baratosen realidad la producción de los mismos ha incurrido en otros muchos gastos que la sociedad pagará no como usuario de la electricidad sino con impuestos como contribuyente. Son costes ambientales no internalizados, son subvenciones directas e indirectas, ayudas históricas como las que otorgaba el marco legal y estable, los costes de transición a la competencia y otras cantidades que no están en el precio de esos kilovatios convencionales.

En febrero de 2002 Comisiones Obreras, Unión General de Trabajadores, Ecologistas en Acción, Greenpeace España y ADENA-WWF rechazan las declaraciones de la Comisión Nacional de Energía (CNE) contrarias a mantener las bonificaciones a la energía eólica. El carácter autónomo de este tipo de energía, su contribución a la reducción de las emisiones de gases de efecto invernadero y el grado de desarrollo tecnológico alcanzado, según estas organizaciones no justifican el freno que se pretende poner a su desarrollo. Por este motivo, solicitan a la CNE que aclare su posición al respecto y realice un informe sobre las ayudas que perciben las distintas fuentes de energía, la demanda de energía eléctrica y gas natural, las emisiones de gases de efecto invernadero de cada uno de ellas y los requisitos necesario para cumplir el Protocolo de Kioto.

La CNE publica en marzo de 2002 el documento Nota en relación a las cuestiones planteadas por CC.OO., UGT, Ecologistas en Acción, Greenpeace España y WWF/ADE$N A$, relacionadas con la CNE, la energía eólica y otras energías. En el resumen se reitera que la CNE ha de contemplar a las energías renovables, al igual que al resto de energías y actividades, bajo el fin básico de la Ley del Sector Eléctrico, cuyos objetivos son garantizar tanto el suministro eléctrico como la calidad de su consumo y que se realice al menor coste posible, sin olvidar la protección del medio ambiente.

Concluye el resumen señalando que «por ello, a las energías renovables que llevan inherentemente asociadas una eficiencia medioambiental positiva, debe permitírsele desde la regulación el desarrollo de unas prestaciones eléctricas más eficientes que las que hasta el momento tienen, para que contribuyan a una mayor garantía y calidad de suministro con el menor coste posible. El mecanismo propuesto por la Comisión para compatibilizar estos objetivos y alcanzar esas prestaciones eléctricas, es incentivar económicamente el acceso voluntario de las energías renovables al mercado de electricidad, teniendo en cuenta los avances tecnológicos recientes y las características especiales de estos medios de producción» (Comisión Nacional de Energía, 2002).

Por tanto, el desarrollo de la energía eólica en España no parece que se vaya a detener por varias razones: la existencia de un potencial significativo; la simplicidad a la hora de su implantación, ya que se trata de instalaciones modulares con equipos iguales y poca obra civil asociada; las posibilidades que ofrece de mejora técnica, aumento de potencia unitaria y reducción significativa de costes; por su reducido impacto ambiental en comparación con otras tecnologías de producción de energía eléctrica; y sobre todo por el importante apoyo político, tanto estatal como de la mayoría de las Comunidades Autónomas. 


\section{Bibliografía}

ALONSO DÍAZ, M. (1993): «La energía eólica. De la investigación a la realidad industrial», en Energía. Revista de Ingeniería Energética, año XIX, nº 6, pp. 153-160.

BARASOAIN, J. A. y FONTÁN, L. (1955): La energía del viento en España y su aprovechamiento. Comisión Nacional de Energía Eólica. Madrid, 41 pp.

BARASOAIN, J. A. y FONTÁN, L. (1962): «Prospección eólica y solar en España.», en Urania, año XLVII, pp. 76-81.

BENAVIDES, P. (1997): «La Política Energética de la Unión Europea», en Economía Industrial, $\mathrm{n}^{\circ}$ 316 , pp. 17-25.

BOLAÑOS, A. (2003): «El proyecto del primer parque eólico español entra en su fase decisiva. El complejo energético, para la costa de Cádiz, prevé una potencia de 1.000 megavatios», en diario El País, 22 de septiembre de 2003, p. 29.

CARDONA, J.L. (1981): Energía eólica y aeroturbinas. Posibilidades de utilización en España. Instituto Nacional de Industria. Madrid, $32 \mathrm{pp}$.

CASTRO GIL, M., COLMENAR SANTOS, A. y SÁNCHEZ NAVARRO, C. (2001): Energía eólica. Promotora General de Estudios S.A. Madrid, 50 pp.

COMISIÓN NACIONAL DE ENERGÍA (2002): Nota en relación a las cuestiones planteadas por CC.OO., UGT, Ecologistas en Acción, Greenpeace España y WWF/ADENA, relacionadas con la CNE, la energía eólica y otras energías. Madrid, $11 \mathrm{pp}$.

DELGADO MARÍN, J.P. ET AL (2003): La energía eólica en la Comunidad Autónoma de la Región de Murcia. Agencia de Gestión de Energía en la Región de Murcia, Murcia, 95 pp.

ESPEJO MARÍN, C. (2001): «El sector eléctrico español en la prensa escrita», en Cuadernos Geográficos de la Universidad de Granada, no 31, pp. 203-217.

ESPEJO MARÍN, C. (2001): «El proyecto de fusión de Endesa e Iberdrola y su desistimiento. Apuntes sobre el sector eléctrico en España», en Nimbus, nº 7-8, pp. 51-65.

FERNÁNDEZ DE SALAMANCA, D. (2001): «Energías renovables. Aprovechamiento de la energía eólica», en Iberdrola. Ponencias Generación. Senda Editorial. Madrid, pp. 95-109.

FONT TULLOT, I. (2000): Climatología de España y Portugal. Universidad de Salamanca. Salamanca, 422 pp.

GARCÍA ORTEGA, J.L. (2003): «Viento en popa. Una propuesta de Greenpeace de Plan Eólico Marino para España», en APPAINFO. Boletín de la Asociación de Productores de Energías Renovables, $\mathrm{n}^{\circ} 13$, pp. 8-11.

GÓMEZ ESPÍN, J.M. (1998): «Primer parque de energía eólica de la Región de Murcia», en Papeles de Geografía, no 28, pp. 171-172.

HERNÁNDEZ NAVARRO, M.L. (1990): «Frecuencia e intensidad del viento en Zaragoza», en Geographicalia, $\mathrm{n}^{\circ}$ 27, pp. 63-75.

INSTITUTO PARA LA DIVERSIFICACIÓN Y AHORRO DE LA ENERGÍA. (1996): Manual de energía eólica. Madrid, $152 \mathrm{pp}$.

INSTITUTO PARA LA DIVERSIFICACIÓN Y AHORRO DE LA ENERGÍA. (2000): Plan de Fomento de las Energías Renovales en España. Madrid, 271 pp.

INSTITUTO PARA LA DIVERSIFICACIÓN Y AHORRO DE LA ENERGÍA. (2003): «Eficiencia energética y energías renovables», en Boletín IDEA, n 5,131 pp.

KASTENDEUCH, P.PH. (2000): «La mesure du vent: conventions, instruments et données», en Revue Géographique de L'Est, tome XL-3, pp. 115-123.

LAALI, A.R. et BERNARD, M. (1999): «Production d'électricité par énergie éolienne. Situation dans le monde et programme français Eole 2005», en La Météorologie, n 25, pp. 38-48.

MARTÍ EZPELETA, A., GARCÍA MARTÍNEZ, E. y MIRAGA VERAS, A. (1998): «Rachas máximas y temporales de viento en Galicia», en Lurralde, nº 21, pp. 221-280.

MARTÍNEZ DE PISÓN, E. (1987): «Las Islas Canarias», en Geografía Regional de España. Ariel. Barcelona, pp. 479-511.

MENÉNDEZ PEREZ, E. (2001): Energías renovables, sustentabilidad y creación de empleo. Una economía impulsada por el sol. Los Libros de la Catarata. Madrid, 270 pp. 
MINISTERIO DE ECONOMÍA (2002): Planificación de los Sectores de Electricidad y Gas. Desarrollo de las Redes de Transporte 2002-2011. Madrid, Secretaría de Estado de Energía.

MINISTERIO DE INDUSTRIA y ENERGÍA (2000): Ley del Sector Eléctrico Disposiciones complementarias. Madrid, $380 \mathrm{pp}$.

MOLINA IBÁÑNEZ, M. (2001): «Las fuentes de energía», en GIL OLCINA, A. y GÓMEZ MENDOZA, J. (Coords.): Geografía de España. Ariel. Barcelona, pp. 455-476.

MORALES GIL, A. (2004): «Significado y valor de las actividades extractivas en la Región de Murcia», en Boletín de la Asociación de Geógrafos Españoles, nº 38, pp. 71-84.

NAVARRO ARANDA, A. y FERNÁNDEZ DE SALAMANCA, D. (2001): «La energía eólica y el desarrollo sostenible», en Iberdrola. Ponencias Generación. Senda Editorial. Madrid, pp. 83-94.

OLCINA CANTOS, J. (1994): Riesgos climáticos en la Península Ibérica. Libros Penthalon-Acción Divulgativa. Madrid, $440 \mathrm{pp}$.

PLAZA BALAGUER, V. (1998): «Respuestas del sector eléctrico a la problemática del efecto invernadero», en Energía y Cambio Climático. Ministerio de Medio Ambiente, Madrid, pp. 97130.

RED ELÉCTRICA DE ESPAÑA (2002): El sistema eléctrico español. Informe 2001. Madrid, 113 pp.

RED ELÉCTRICA DE ESPAÑA (2003): El sistema eléctrico español. Informe 2002. Madrid, 105 pp.

SANZ MENDIOLA, I. y CALVO CASTRO, C.L. (1997): «La energía como actividad económica en España. El caso particular del sector eléctrico», en Economía Industrial, nº 316, pp. 199-217.

SEMPERE, P. y CANO, J.L. (1982): «Una evaluación de la energía eólica disponible en la zona costera de La Coruña», en Revista de Geofísica, n ${ }^{\circ}$, pp. 73-79.

UNESA (1998): Centrales eléctricas. Madrid, $71 \mathrm{pp}$.

UNESA (2001): La industria eléctrica y el medio ambiente. Madrid, 159 pp.

VIEDMA MUÑOZ, M. (1983): «La presión atmosférica y los vientos en Almería», en Paralelo 37, $n^{\circ}$. 7, pp. 83-92.

VIEDMA MUÑOZ, M. (1999): «Análisis de las direcciones de los vientos en Andalucía». en Nimbus, $\mathrm{n}^{\circ} 1-2$, pp. 153-168. 
